\title{
A Micro-Doppler Frequency Ambiguity Resolution Method Based on Complex-Valued U-Net
}

\author{
Ming Long $\mathbb{B}^{D}$, Jun Yang, Saiqiang Xia, Mingjiu Lv, Xu Wei, and Wenfeng Chen
}

Early Warning Academy, Wuhan 430019, China

Correspondence should be addressed to Ming Long; alphaergo@163.com

Received 3 May 2021; Revised 27 June 2021; Accepted 13 July 2021; Published 21 July 2021

Academic Editor: Li Haitao

Copyright (c) 2021 Ming Long et al. This is an open access article distributed under the Creative Commons Attribution License, which permits unrestricted use, distribution, and reproduction in any medium, provided the original work is properly cited.

In order to resolute the micro-Doppler frequency ambiguity caused by radar pulse repetition frequency not high enough (i.e., pulse dimension does not satisfy the requirement of Nyquist sampling theorem), this paper presents a micro-Doppler frequency ambiguity resolution method based on complex-valued U-net. The echo sequence is interpolated by zeros in the pulse dimension to increase the equivalent pulse repetition frequency, so that the echo sequence after zero interpolation contains the real microDoppler frequency; at the same time, some new frequency components are generated. The variation law of the echo sequence frequency after zero interpolation is analyzed. Then, the echo sequence in time domain after zero interpolation is transformed to the time-frequency domain by short-time Fourier transform (STFT). Finally, the time-frequency results can be segmented by the model, which is trained by complex-valued U-net to eliminate the redundant frequencies generated by zero interpolation; thus, the reconstruction of real micro-Doppler frequency is realized. Theoretical analysis and simulation results show that the proposed method can solve the problem of micro-Doppler frequency ambiguity. Compared with fully convolution network (FCN) and fully convolution residual network (FCRN), the proposed method has better performance and robustness.

\section{Introduction}

In modern warfare, helicopter has the advantages of vertical take-off and landing and strong transportation capabilities. It has become the preferred equipment for military operations such as ground attack, weapon delivery, and strategic support $[1,2]$. At the same time, helicopter has become one of the main targets of air defense operations in various countries. When this kind of target is hovering or flying at a slow speed, it is difficult to meet the requirements of detection and recognition by using traditional means, so it is particularly important to use micromotion characteristics for feature extraction and recognition [3-5]. However, for helicopter with fast rotation speed or long length, when the radar pulse repetition frequency cannot satisfy the Nyquist sampling theorem, the phenomenon of micro-Doppler frequency ambiguity will occur, which makes the feature extraction, parameter estimation, and recognition of rotor targets more difficult.
For modern radar, especially pulse-Doppler (PD) radar, it usually adopts different working modes $[6,7]$ to deal with different tasks. The main working modes are low pulse repetition frequency (LPRF) mode [8], medium pulse repetition frequency (MPRF) mode [9-11], and high pulse repetition frequency (HPRF) mode $[12,13]$. However, range ambiguity or doppler ambiguity exists in different working modes. In view of this kind of problems, a lot of researches have been carried out on ambiguity resolution algorithms. At present, the methods of doppler ambiguity resolution are usually to form a group of pulse repetition frequency with multiple pulse repetition frequencies and then complete ambiguity resolution on this basis. The main algorithms include one-dimensional set algorithm [13], Chinese remainder theorem [14, 15], and look-up table method $[16,17]$. Compared with the traditional doppler frequency ambiguity, the micro-Doppler frequency ambiguity is different. From the mechanism point of view, the microDoppler frequency is generated by the rotating parts of the target rather than the radial motion of the target. In addition, 
the relationship between traditional Doppler frequency and phase is generally linear, while the micro-Doppler frequency and phase satisfy the sinusoidal modulation relationship [18-20].

As a machine learning method [21-23], deep learning will classify and recurse according to the input data. Deep learning is mainly realized by neural network, which is an extensive, parallel, and interconnected network composed of adaptive simple units. Its structure can simulate the interaction of biological neural system to real world objects [24-28]. The deep learning algorithm used in this paper is U-net [29]. U-net is a symmetric coded-decoding network, which fuses the feature map generated by the coding layer with the feature map of the corresponding decoding layer to generate the final feature map of the decoding layer. U-net is mainly composed of convolution layers, pooling layers (downsampling layers), and upsampling layers and uses residual network for feature extraction with strong extraction performance. Because U-net uses convolution layers to replace the fully connected layer in convolutional neural network, the network carries out symmetrical coding and decoding, which causes the output vector of the network to be related to the input vector. Therefore, U-net has good applications in target segmentation, semantic segmentation, and target detection, especially in the field of image segmentation [30-32].

In this paper, aimed at the problem of micro-Doppler frequency ambiguity, a method of micro-Doppler frequency ambiguity resolution based on complex-valued U-net and zero interpolation is proposed. Theoretical analysis is focused on the reason of micro-Doppler frequency generation, and the change rule of micro-Doppler frequencies of echo sequence after zero interpolation. Because the pulse repetition frequency of the echo sequence is increased after zero interpolation, the echo sequence contains real microDoppler frequencies, and some redundant frequencies are also generated. Then, the time-frequency results of the novel echo sequence are obtained by using STFT. The ambiguous time-frequency results corresponding to the micromotion sequence after zero interpolation are used as the input of the U-net network, and the unambiguous time-frequency results corresponding to the micromotion sequence after expanding the pulse repetition frequency are used as the labels of the U-net. The U-net is extended to the complex domain to train the model and generate the training model. Finally, compared with the fully convolution network (FCN) and fully convolution residual network (FCRN), the complex-valued U-net is used to eliminate the redundant frequency components to reconstruct the real micro-Doppler frequency. Theoretical analysis and simulation results show that the proposed method is feasible and robust and can solve the problem of micro-Doppler frequency ambiguity.

\section{Analysis of Micro-Doppler Frequency Characteristics of Helicopter}

2.1. Echo Micro-Doppler Frequency Analysis. Figure 1 is the geometric relationship diagram between radar and rotor blades. According to $[19,20]$, different blades have the same

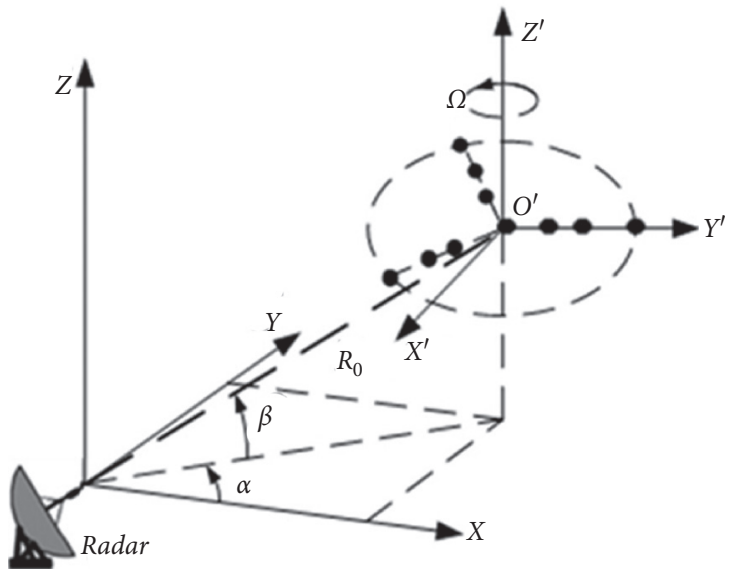

FIGURE 1: The geometric relationship diagram between radar and rotor blades.

number of scattering points with the same interval and scattering coefficient. At this time, the echo of the rotor blades scattering point model can be expressed as

$$
{ }^{s} \sum^{(t)}=\sum_{n=1}^{N} s_{n}(t)=e^{\left(-j\left(4 \pi R_{0} / \lambda\right)\right)} \sum_{n=1}^{N} \sum_{i=1}^{K} \sigma_{P_{i}} e^{f_{d P_{i, n}}(t)},
$$

where $\sigma_{P_{i}}$ is the backscattering coefficient, $R_{0}$ is the distance between the radar and the rotor center $O I$, and $f_{d P_{i n}}(t)$ is the doppler frequency caused by the $i$ th scattering point on the $n$th blade, as follows:

$$
f_{\mathrm{d} P_{i, n}}(t)=\frac{4 \pi f_{\mathrm{rot}} x_{i}}{\lambda} \sin \left(\theta_{1}+\frac{(n-1) 2 \pi}{N}+2 \pi f_{\mathrm{rot}} t\right) \cos \beta,
$$

where $\beta$ is the pitch angle, $f_{\text {rot }}$ is the rotor rotation frequency, $x_{i}(0 \leq x \leq l, l$ is the blade length) is the distance between the scattering point $P_{i}$ and the rotor center, and $\theta_{1}$ is the initial rotation angle.

$$
f_{\mathrm{d} P_{i, n}}(t)=\frac{4 \pi f_{\mathrm{rot}} x_{i}}{\lambda} \sin \left(\theta_{1}+\frac{(n-1) 2 \pi}{N}+2 \pi f_{\mathrm{rot}} t\right) \cos \beta .
$$

According to equation (3), the peak Doppler frequency is closely related to radar wavelength, rotor rotation frequency, blade length, and pitch angle. At the same time, in order to ensure that the Doppler frequency is unambiguous, the pulse repetition frequency (sampling frequency on pulse dimension) needs to meet the requirements.

$$
\mathrm{PRF} \geq 2 f_{d \max }(t)=\frac{8 \pi f_{\text {rot }} l}{\lambda} \cos \beta .
$$

Equation (4) can be called the lower limit formula of PRF that the radar needs to meet when detecting the echo of the rotor blades. From the analysis of equation (4), the peak Doppler is proportional to the rotor rotation frequency, blade length, and pitch angle. The radar wavelength is determined by the radar carrier frequency. The higher the radar carrier frequency is, the greater the peak Doppler frequency is. Figure 2 shows the time-frequency results of 


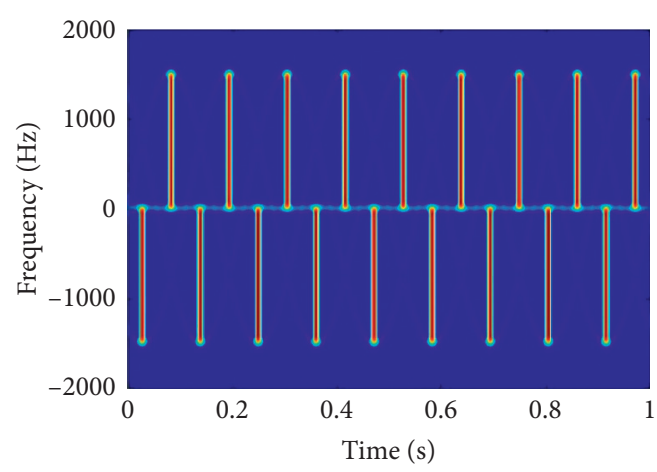

(a)

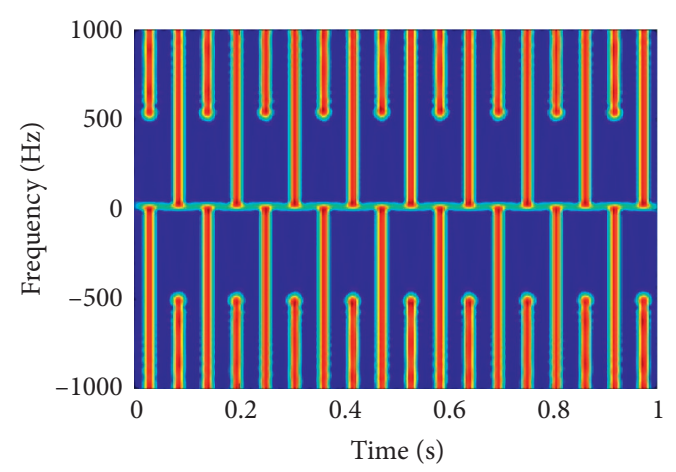

(b)

FIGURE 2: Influence of PRF change on time-frequency result. The time-frequency result of echo sequence when PRF (a) meets the lower bound formula and (b) does not meet the lower bound formula.

the echo sequence when the PRF value does not meet $(1000 \mathrm{~Hz})$ and meets $(2000 \mathrm{~Hz})$ the PRF lower limit formula. Through comparison, it is found that when the PRF value does not meet the PRF lower limit formula, the spectrum aliasing will occur, that is, micro-Doppler frequency ambiguity. Therefore, in the analysis of the rotor target, it is necessary to select the appropriate PRF value to avoid the adverse effects of frequency ambiguity.

2.2. Analysis of the Influence of Zero Interpolation on the Frequency Component of Signal. Aiming at micro-Doppler frequency ambiguity, the method of sampling signal interpolation by zeros is equivalent to enlarging its sampling frequency, so that the processed signal satisfies Nyquist sampling theorem, and the real frequency appears in the spectral analysis, thus providing a new method for resolving spectrum aliasing. By this method, the echo sequence can be written as

$$
x(n)=\sum_{i=1}^{K} a_{i} e^{\left(j 2 \pi f_{i} n t_{r}\right)}, t_{r}=\frac{1}{f_{s}}, \quad i=1,2,3, \ldots, K .
$$

where $t_{r}$ is the sampling period, $f_{s}$ is the sampling frequency, $f_{s} \geq 2 \cdot \max \left\{f_{1}, f_{2}, \ldots, f_{K}\right\}$, the frequency range after sampling is $\left[\left(-f_{s} / 2\right),\left(f_{s} / 2\right)\right]$, and the bandwidth is $f_{s}$. Interpolating zeros to the echo sequence means that the sampling frequency changed. At the same time, it also changes the frequency information contained in the echo sequence and makes the sequence show new frequency components. After interpolating Mmultiples of zeros, the signal satisfies Nyquist sampling, and the signal can be expressed as

$$
x_{1}(n)=\left\{\begin{array}{l}
e^{\left(j 2 \pi f_{1} n t_{r}\right)}, t_{r}=\frac{1}{f_{s}}, f_{1} \leq \frac{f_{s}}{2}, \quad n=(M+1) p+1 \\
0, n=(M+1) p+2,(M+1) p+3, \ldots, \quad(M+1)(p+1) \quad p=0,1, \ldots, N .
\end{array}\right.
$$

The method of interpolating zeros is shown as above, that is, interpolating zeros with equal interval and equal number in the middle of the original sequence to increase the sampling frequency by an integral multiple. Through theoretical derivation, it can be found that the original sampling sequence is equivalent to adding $M$ new frequency component after interpolating $M$ multiples zeros, the energy of the signal is evenly distributed to each frequency component, and the amplitude of each frequency component becomes $1 / M+1$ before zero interpolation. At this time, the sampling frequency is equivalent to $f_{s M}=(M+1) f_{s}$, and the frequency component is shown in Table 1.

It can be seen from Table 1 that, for the undersampled echo sequence, we can make the sampling frequency meet the Nyquist sampling theorem by interpolating zeros in the signal processing, and then we can get the real frequency of the signal. In this paper, STFT is performed after interpolating zeros to the echo sequence of helicopter with three rotor blades, as shown in Figure 3. Compared with Figure 2, it can be found that, for the time-frequency results of rotor targets with frequency ambiguity, the real frequency value of the ambiguous spectrum component is shown in the timefrequency results through the zero interpolation, and the spectrum distribution is more uniform, which provides favorable conditions for the next step of image segmentation by using U-net.

\section{Complex-Valued U-Net}

Different from the conventional image data set commonly used in image segmentation, grey image and RGB threechannel image are used as input in training. In this paper, 
TABLE 1: Frequency components after zero interpolation.

\begin{tabular}{lc}
\hline Interpolating zero multiples $M$ & Including frequency component \\
\hline$M=0$ & $f_{1}$ \\
$M=1$ & $f_{1}, f_{1}-f_{s}$ \\
$\vdots$ & \\
$M=2 q$ & $f_{1}, f_{1}-f_{s}, f_{1}+f_{s}, \ldots, f_{1}-q f_{s}, f_{1}+q f_{s}$ \\
$M=2 q+1$ & $f_{1}, f_{1}-f_{s}, f_{1}+f_{s}, \ldots, f_{1}+q f_{s}, f_{1}-(q+1) f_{s}$ \\
\hline
\end{tabular}

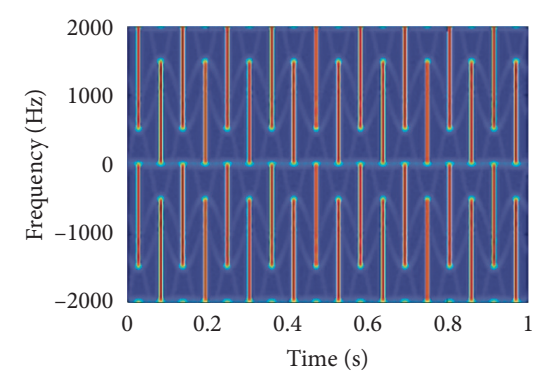

(a)

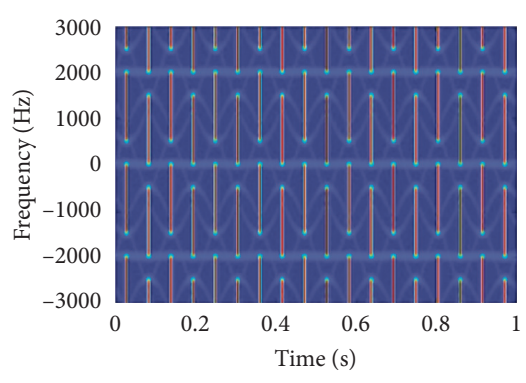

(b)

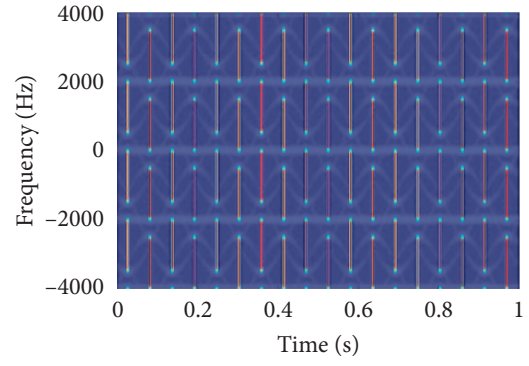

(c)

Figure 3: Time frequency results of echo sequence after zero interpolation. The time-frequency result of echo sequence after interpolating (a) once of zeros, (b) double of zeros, and (c) triple of zeros.

STFT is used to generate the time-frequency result of the signal after the zero interpolation. Any point on the timefrequency result is complex-valued, which is composed of real part and imaginary part. For radar echo, both the real part and the imaginary part contain useful information. In order to retain more useful information, U-net is used in the complex-valued, and the time-frequency result is divided into real channel and imaginary channel. Then, the output data of the two channels are combined into a complex-value to realize image segmentation and remove the redundant part of the time-frequency result.

3.1. Design of Complex-Valued U-Net. This paper uses a complex domain U-net, and the architecture is shown in Figure 4.

The input of the network is a two-dimensional complex time-frequency result after zero interpolation. Any point on the instant frequency result is a complex number. For discrete echo sequences, STFT is used here to generate a $400 \times 400$ matrix time-frequency result, with each point in the time-frequency result set to

$$
P_{c z}=a_{c z}+i b_{c z}
$$

In the equation, $c=1,2,3, \ldots, 400, z=1,2,3, \ldots, 400$.

Firstly, we take the modulus to find the maximum value as

$$
P_{\max }=\max \left\{\sqrt{a_{c z}^{2}+b_{c z}^{2}}\right\}
$$

Then, we separate the real part and the imaginary part, and the real part matrix is

$$
R_{c z}=\frac{a_{c z}}{P_{\max }}=\left[\begin{array}{cccc}
\frac{a_{1,1}}{P_{\max }} & \cdots & \cdots & \frac{a_{1,400}}{P_{\max }} \\
\vdots & \ddots & \ddots & \vdots \\
\vdots & \ddots & \ddots & \vdots \\
\frac{a_{400,1}}{P_{\max }} & \cdots & \cdots & \frac{a_{400,400}}{P_{\max }}
\end{array}\right] .
$$

The imaginary part matrix is

$$
F_{c z}=\frac{b_{c z}}{P_{\max }}=\left[\begin{array}{cccc}
\frac{b_{1,1}}{P_{\max }} & \cdots & \cdots & \frac{b_{1,400}}{P_{\max }} \\
\vdots & \ddots & \ddots & \vdots \\
\vdots & \ddots & \ddots & \vdots \\
\frac{b_{400,1}}{P_{\max }} & \cdots & \cdots & \frac{b_{400,400}}{P_{\max }}
\end{array}\right] .
$$

These two matrices are used as two channels input data for encoding and decoding operations in a complex U-net. The U-net in this paper has seven layers, and the input and output time-frequency result sizes are $400 \times 400$. The network training steps are as follows:

(1) The time-frequency result of the input data set is processed. The time-frequency result is divided into real part matrix and imaginary part matrix. The matrix is processed according to equation (9) and equation (10) as the input of the network. 


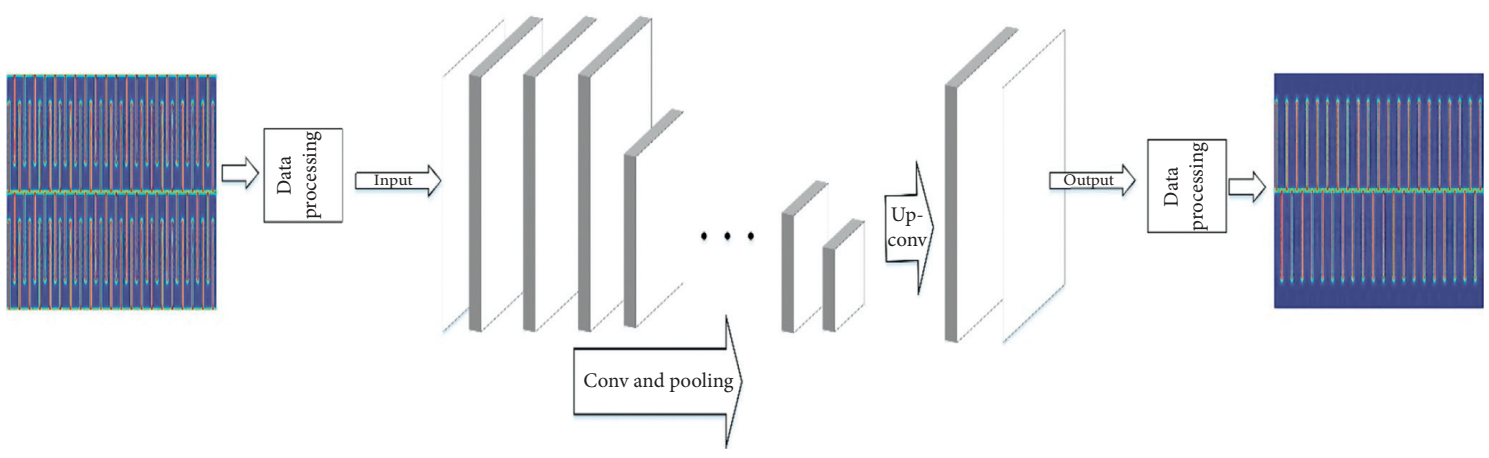

FIgURE 4: The architecture of our complex-valued U-net.

(2) The first four layers are convolution and pooling, which compresses the data and extracts some simple features. The last three layers are upsampling and convolution. Some deeper features can be obtained through upsampling convolution. Through the jump structure, the data of the first three layers can be combined with the data of the last three layers and get better image segmentation performance by combining with the data of the deep layer. At last, the image segmentation is realized by the $1 \times 1$ convolution kernel of the seventh layer. The structure and operation flow of complex-valued U-net are shown in Figure 5.

The network structure is as follows:

Layer 1: conv, filters: 16 , kernel_size: $3 \times 3$, strides: 1 , $\mathrm{BN}$, activation function: ReLU

Conv, filters: 16 , kernel_size: $3 \times 3$, strides: $1, \mathrm{BN}$, activation function: ReLU

Conv, filters: 16 , kernel_size: $3 \times 3$, strides: $1, \mathrm{BN}$, activation function: $\operatorname{ReLU}$

Layer 2: max pooling, filters: 16 , pool_size: $2 \times 2$, strides: 2

Conv, filters: 32, kernel_size: $3 \times 3$, strides: $1, \mathrm{BN}$, activation function: ReLU

Conv, filters: 32, kernel_size: $3 \times 3$, strides: 1 , BN, activation function: ReLU

Layer 3: $\max$ pooling, filters: 32, pool_size: $2 \times 2$, strides: 2

Conv, filters: 64 , kernel_size: $3 \times 3$, strides: $1, \mathrm{BN}$, activation function: ReLU

Conv, filters: 64 , kernel_size: $3 \times 3$, strides: $1, \mathrm{BN}$, activation function: ReLU

Layer 4: $\max$ pooling, filters: 64 , pool_size: $2 \times 2$, strides: 2

Conv, filters: 128 , kernel_size: $3 \times 3$, strides: $1, \mathrm{BN}$, activation function: ReLU

Conv, filters: 128 , kernel_size: $3 \times 3$, strides: $1, \mathrm{BN}$, activation function: ReLU

Layer 5: upconv, filters: 64, kernel_size: $3 \times 3$, strides: 2 , $\mathrm{BN}$, activation function: ReLU
Copy and crop: layer 3 and layer 5

Conv, filters: 64 , kernel_size: $3 \times 3$, strides: $1, \mathrm{BN}$, activation function: $\operatorname{ReLU}$

Conv, filters: 64 , kernel_size: $3 \times 3$, strides: $1, \mathrm{BN}$, activation function: ReLU

Layer 6: upconv, filters: 32, kernel_size: $3 \times 3$, strides: 2 , $\mathrm{BN}$, activation function: ReLU

Copy and crop: layer 4 and layer 6

Conv, filters: 32 , kernel_size: $3 \times 3$, strides: $1, \mathrm{BN}$, activation function: ReLU

Conv, filters: 32 , kernel_size: $3 \times 3$, strides: $1, \mathrm{BN}$, activation function: ReLU

Layer 7: upconv, filters: 16 , kernel_size: $3 \times 3$, strides: 2 , $\mathrm{BN}$, activation function: ReLU

Copy and crop: layer 1 and layer 7

Conv, filters: 16 , kernel_size: $3 \times 3$, strides: 1 , BN, activation function: ReLU

Conv, filters: 16 , kernel_size: $3 \times 3$, strides: 1 , BN, activation function: ReLU

Conv, filters: 2, kernel_size: $1 \times 1$, strides: 1

Copy and crop: layer 1 and layer 7

In this network structure, Conv is convolution, $\mathrm{BN}$ is batch normalization, upconv is upconvolution for upsampling, and the activation function is ReLU, which is called rectified linear unit shown in the following equation:

$$
\operatorname{ReLU}(x)= \begin{cases}x, & \text { if } x>0 \\ 0, & \text { if } x \leq 0\end{cases}
$$

\subsection{Save the Training Model}

3.2.1. Design of Training Model. The ambiguous time-frequency results corresponding to the micromotion signal after zero interpolation and the unambiguous time-frequency results corresponding to the micromotion signal after expanding the pulse repetition frequency are taken as the input and the label of the dataset, respectively. Then, the complex-valued U-net is trained to generate the training model and compared with the full convolution network (FCN) and full convolution residual network (FCRN). Then, 


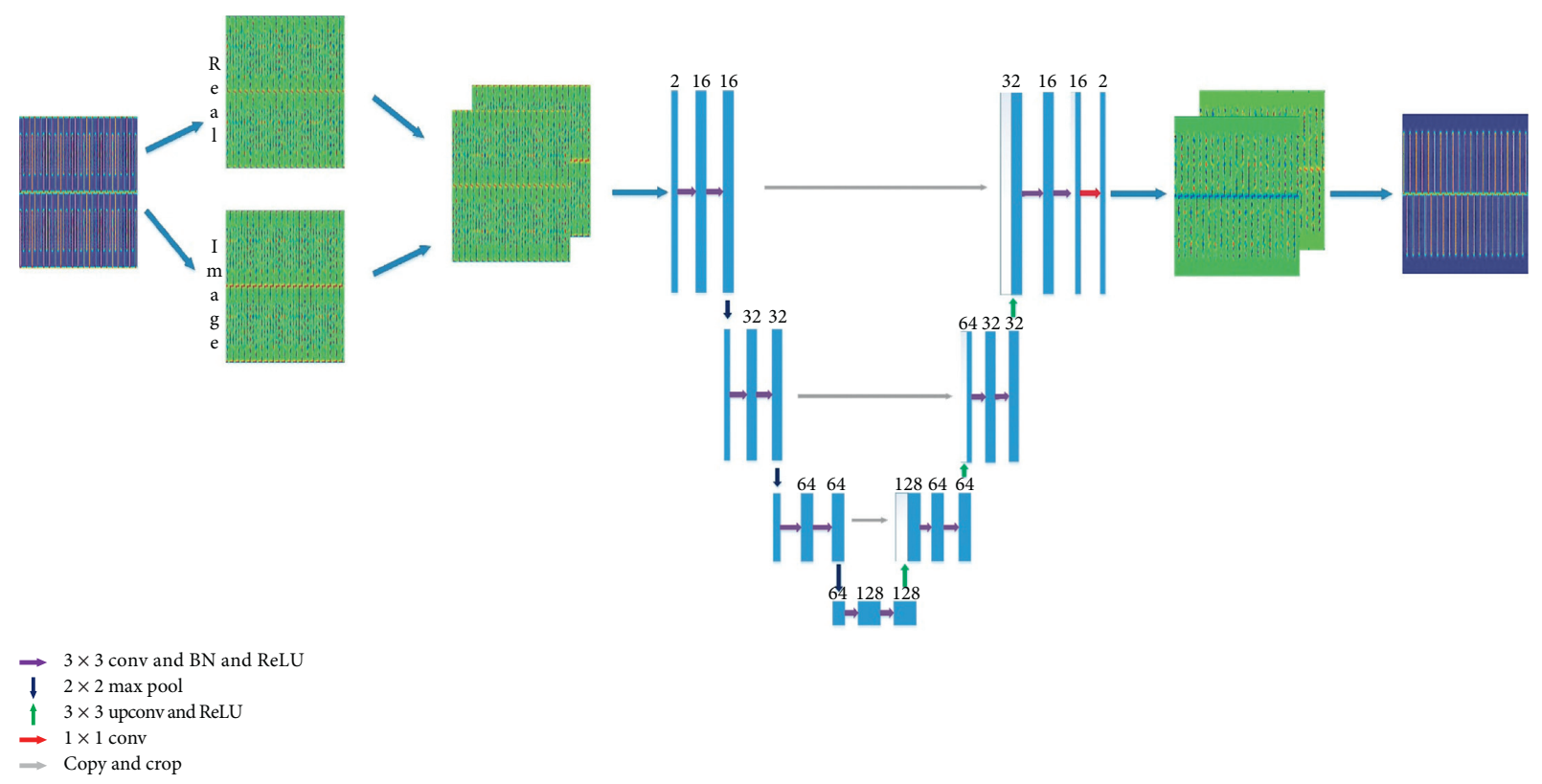

FIGURE 5: The structure and operation flow of complex-valued U-net.

the performance of the designed U-net is tested. The training model flow is shown in Figure 6.

The training environment configuration and hardware are shown in Table 2.

\section{Dataset}

According to equation (2), there are many factors that affect the time-frequency results of the rotor targets' echo sequence, such as the number of rotor blades, rotational speed, initial phase, and signal-to-noise ratio (SNR). In this paper, the method of controlling variables, setting parameters, and generating data sets are used for training. The initial phase of the echo is random. According to the number of blades, the range of the initial phase satisfies phase $\Phi \subset[0,2 \pi / N]$ and $N$ is the number of blades. The carrier frequency $f_{c}=1 \mathrm{GHzand}$ the wavelength $\lambda=0.3 \mathrm{~m}$. The rotational speed and blade length are set according to the value range of the highest frequency. The performance of the network model is analyzed under different interpolating zero multiples, different number of blades, and different SNR.

4.1. Different Interpolating Zero Multiples. For zero interpolation, the number of interpolating zeros directly affects the number of frequency components and the magnitude of the spectrum. Based on the target echo of three blades rotor targets, the highest frequency in the echo is set to $1000 \mathrm{~Hz} \sim 2000 \mathrm{~Hz}, 2000 \mathrm{~Hz} \sim 3000 \mathrm{~Hz}, 3000 \mathrm{~Hz} \sim 4000 \mathrm{~Hz}$, and the sampling frequency is set to $2000 \mathrm{~Hz}$. The original echo sequence is interpolated by the zero interpolation, and the time-frequency result is generated by using STFT as the input data. Increase the sampling frequency to $4000 \mathrm{~Hz}, 6000 \mathrm{~Hz}, 8000 \mathrm{Hzand}$ the time-frequency results after STFT as output training labels for training. The specific

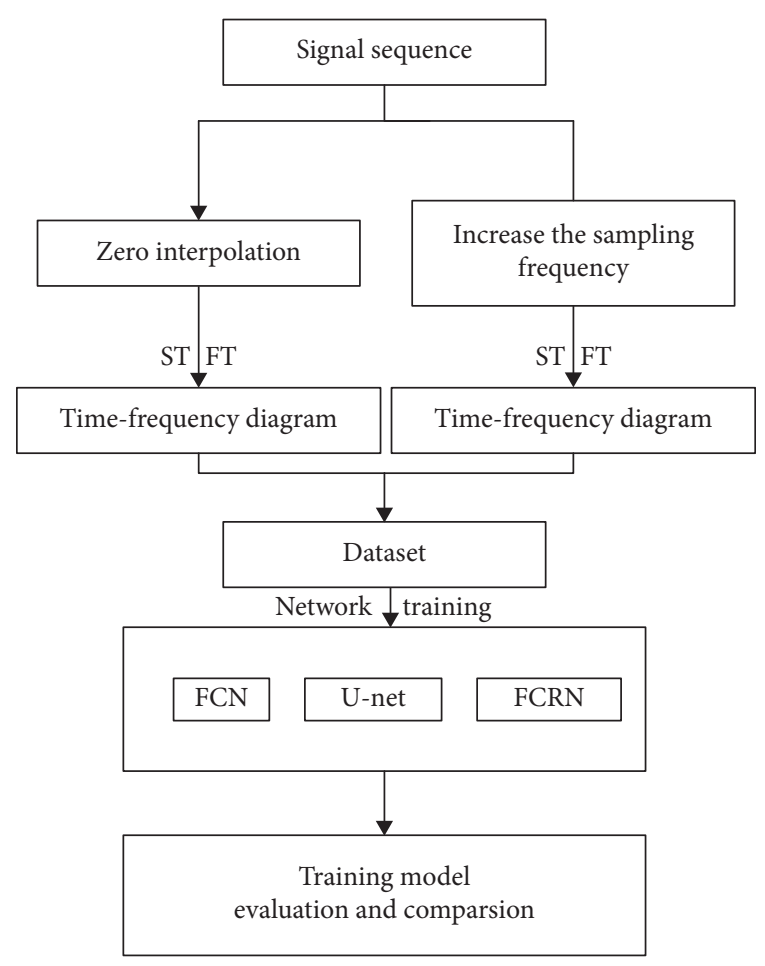

Figure 6: Training model flow.

TABLE 2: Configuration of deep learning platform.

\begin{tabular}{lc}
\hline Configuration & Content \\
\hline System & Windows7 \\
Framework of deep learning & tensorflow1.12.0 \\
GPU & Tesla T4 \\
CPU & Intel core i7 $2.8 \mathrm{GHz}$ \\
\hline
\end{tabular}


parameter settings are shown in Table 3. The input timefrequency results and the output training label time-frequency results are shown in Figures 7 and 8.

4.2. Different Number of Blades. For rotor targets, when the number of rotor blades is different, the characteristics of time-frequency results are different. Parameters are set to make the rotational speed of the blade $5 \sim 7.5 \mathrm{rad} / \mathrm{sand}$ the length of the rotator $5-7.5 \mathrm{~m}$. The highest frequency in the echo sequence is $1000 \mathrm{~Hz} \sim 2000 \mathrm{~Hz}$, and the sampling frequency is $2000 \mathrm{~Hz}$.By using the zero interpolation, the sampling frequency is expanded to $4000 \mathrm{~Hz}$, and the timefrequency result is generated by using STFT as the input data of the U-net. The time-frequency result generated by STFT after increasing the sampling frequency to $4000 \mathrm{Hzis}$ as an output training label. The input time-frequency results and the output training label time-frequency results are shown in Figure 9 and Figure 10.

4.3. Different Signal-to-Noise Ratios. For radar echo, the time-frequency result after STFT is not so clear, due to the presence of noise. The initial parameters under different SNR are used to generate dataset by using parameters in Section 4.2. An input time-frequency result and an output training label are shown in Figure 11.

4.4. Composition of Dataset. The dataset consists of the training set and the test set. The training set is composed of three parts, the data set of different interpolating zero multiples, the data set of different number of rotor blades, and the data set of different SNRs. The composition of the test set is the same as the data set. The composition of training set is shown in Table 4, and the composition of test set is shown in Table 5 .

\section{Simulation Results and Analysis}

5.1. Network Performance Comparison. Three full convolution neural networks are used to compare the performance including FCN, FCRN, and U-net. Figure 12 shows the loss curves of the three networks during training. By comparing the loss curves of three networks, we can find that the loss of FCN and FCRN is much greater than the loss of U-net. With the increase of training cycles, the loss of U-net tends to be stable and less. For the other two networks, the loss decreases with the training cycles, but the fluctuation of the network is violent, which indicates that the training effect and robustness of the U-net model are the best.

Enter a picture of the test set and input three network training models to compare the output time-frequency results. From the results, we can find that the FCN and FCRN of image segmentation performances are extremely poor; not only do they not remove the extra flicker components, but also they blur the original time-frequency results, making it more difficult to extract useful information. The U-net can effectively remove redundant flickers while retaining the required flicker and envelope information.
Table 3: Parameter settings.

\begin{tabular}{lcc}
\hline $\begin{array}{l}\text { Inter } \\
\text { polating multiples }\end{array}$ & $\begin{array}{c}\text { The } \\
\text { speed of the blade }\end{array}$ & The length of the rotator \\
\hline 1 & $5 \sim 7.5 \mathrm{rad} / \mathrm{s}$ & $5 \sim 7.5 \mathrm{~m}$ \\
2 & $6 \sim 8 \mathrm{rad} / \mathrm{s}$ & $6 \sim 8 \mathrm{~m}$ \\
3 & $7 \sim 9 \mathrm{rad} / \mathrm{s}$ & $9 \sim 12 \mathrm{~m}$ \\
\hline
\end{tabular}

Figure 13 shows the results of the same input picture test with three network training models.

5.2. Impact Analysis of Zero Interpolation on U-Net Performance. Figure 14 is training loss curves of the relationship between training rounds with different interpolating zeros multiples. In Figure 14, fs $2 / \mathrm{fs} 3 / \mathrm{fs} 4$ means interpolating once/double/treble of zeros. By comparing the loss curves of different zero interpolating multiples, we can see that, with the increasing of training cycles, the loss rate decreases and finally tends to be stable. However, with the increasing of interpolation zeros, the parameter quantity of time-frequency results increases, the parameter quantity of network model increases, the range value of loss tends to increase stably, and loss tends to fluctuate more easily.

As the number of interpolating zero multiples increases, there is a greater difference in the details of the output results, the number of interpolation zeros increases, the envelope part of the flicker is fragile, and the envelope part of the package flicker is ambiguous. Figure 15 is the outputs after the part of test set using the training model.

The simulation results show that the zero interpolation and the complex-valued U-net model can remove the problem of micro-Doppler ambiguity due to sampling frequency limitation of the target echo, which creates the possibility for the next step in parameter estimation and feature extraction.

5.3. Performance Analysis of Complex-Valued U-Net. Due to the more zeros interpolated by the zero interpolation, the amount of data became larger, and the network model image segmentation effect will be affected. In this paper, the U-net model is analyzed by using the echo sequences with zero interpolation of once of zeros. Performance analysis is divided into two parts: one is performance analysis under different SNRs, and the other is performance analysis under different number of rotor blades.

5.3.1. Different Interpolating Performance Analysis under Different SNR. For the complex-valued U-net, the test set is tested and validated with the generated training model when it performs performance analysis under different SNRs. The input parts of the test set are shown in Figure 16, and the output results are shown in Figure 17. Due to the SNR range, the training set and the test set in this study select $0 \mathrm{~dB}, 3 \mathrm{~dB}, 10 \mathrm{~dB}$ for testing. The result shows that the flicker does not change much with the increase of SNR, but the envelope becomes weaker with the decrease of SNR. The envelope is more obvious than the input of test set, and there is still some loss compared to the label of test set. Through 


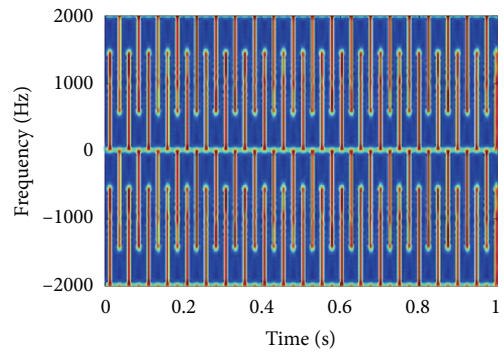

(a)

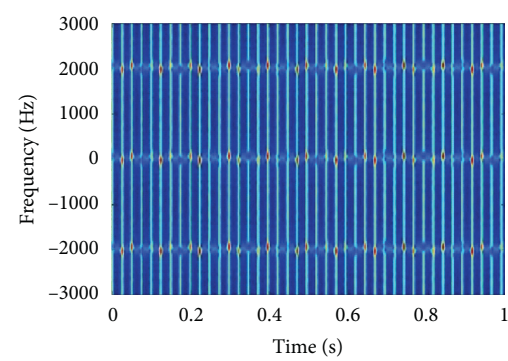

(b)

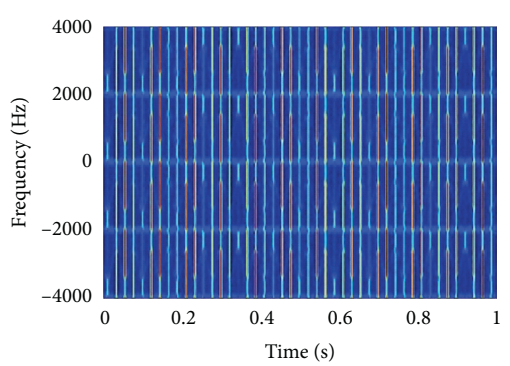

(c)

Figure 7: The input time-frequency results after interpolating different multiples of zero. The time-frequency result interpolating (a) once of zeros, (b) double of zeros, and (c) triple of zeros.

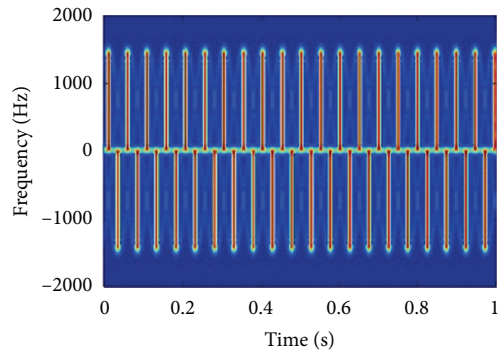

(a)

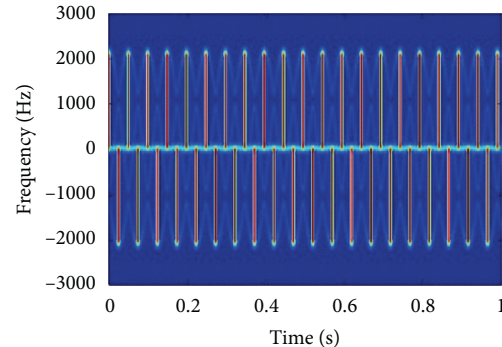

(b)

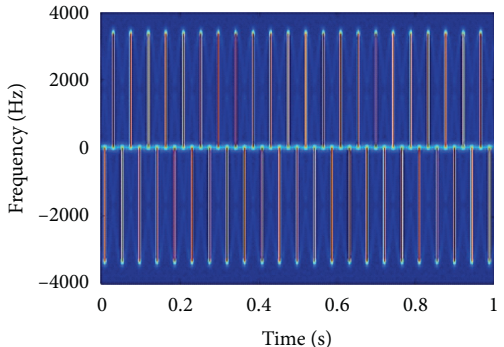

(c)

FIGURE 8: The output training label time-frequency results. Training label of echo sequence after interpolating (a) once of zeros, (b) double of zeros, and (c) triple of zeros.

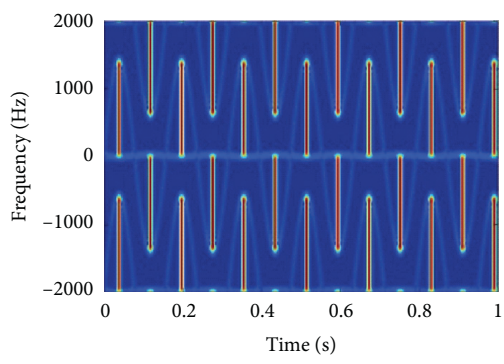

(a)

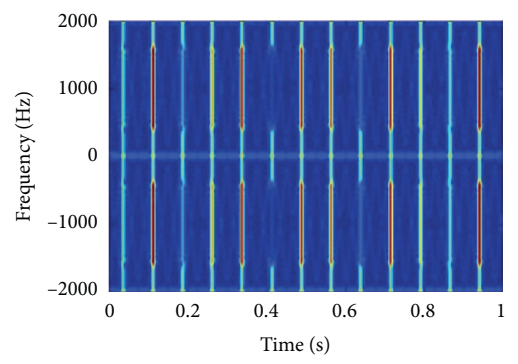

(b)

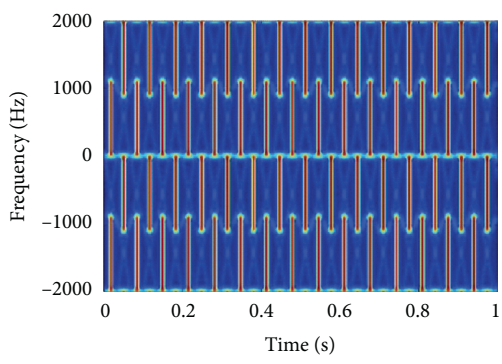

(c)

Figure 9: The input time-frequency results of echo sequence of different rotors. (a) One rotor. (b) Two rotors. (c) Three rotors.

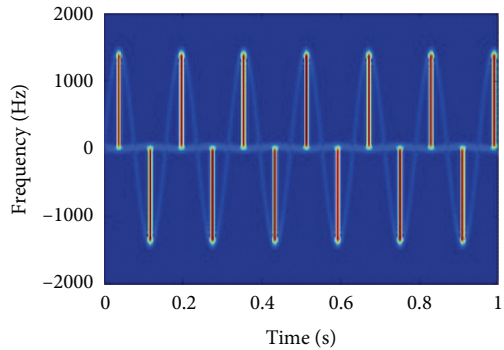

(a)

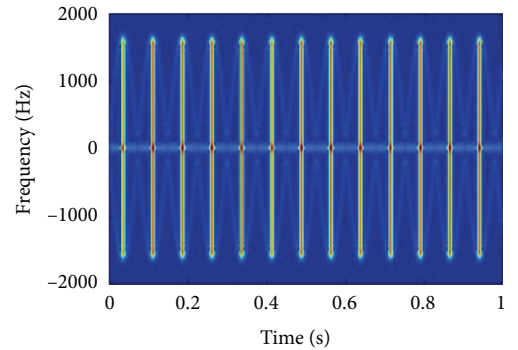

(b)

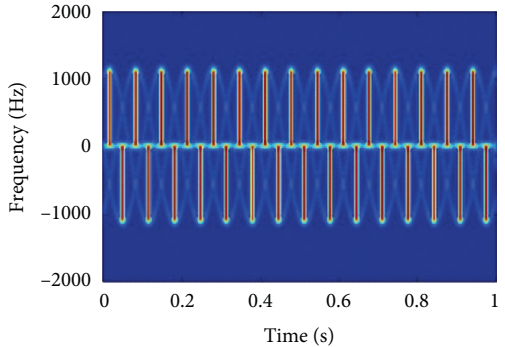

(c)

FIGURE 10: The output training label time-frequency results of echo sequence of (a) one rotor, (b) two rotors, and (c) three rotors.

comparison, it is found that the complex-valued U-net has better performance under different SNRs and can effectively solve the problem of frequency ambiguity in the case of noise.
5.3.2. Performance Analysis under Different Number of Rotor Blades. For rotor targets, the number of rotor blades is different, but the time-frequency characteristics are very 


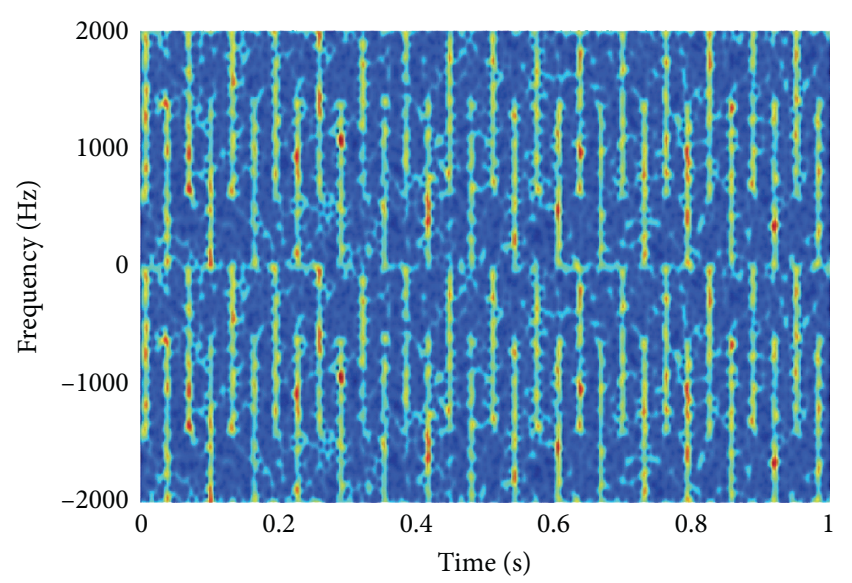

(a)

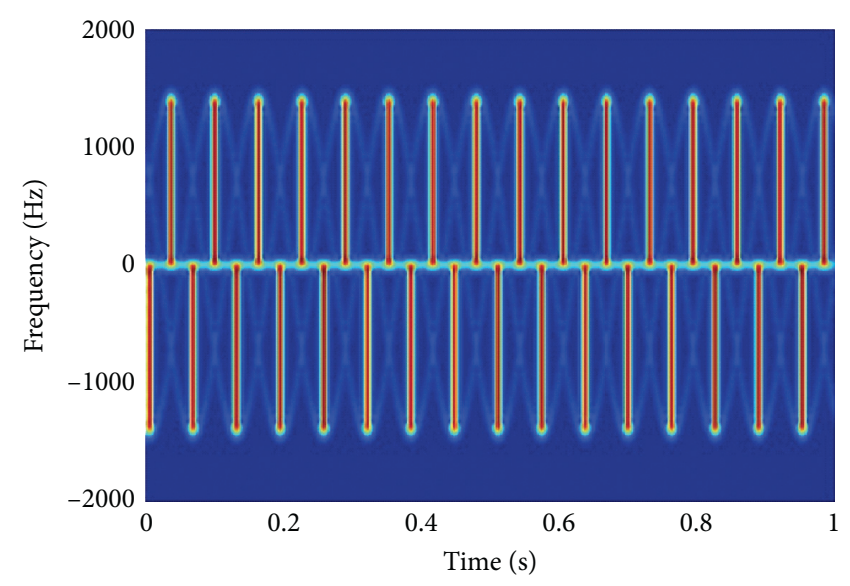

(b)

FIgURE 11: The input time-frequency results with $\mathrm{SNR}=2.875 \mathrm{~dB}$. (a) The time-frequency result. (b) The training label.

TABLE 4: Composition of training set.

\begin{tabular}{|c|c|c|c|}
\hline Data set & Training set & Size & Classes \\
\hline \multirow{3}{*}{ Different interpolating zero multiples } & \multirow{3}{*}{$1000 / 1000 / 1000$} & $400 \times 400$ & 1 \\
\hline & & $600 \times 600$ & 2 \\
\hline & & $800 \times 800$ & 3 \\
\hline Different number of blades & $200 / 200 / 200 / 200 / 200 / 200$ & $400 \times 400$ & $1 / 2 / 3 / 4 / 5 / 6$ \\
\hline Different signal-to-noise ratio & 1000 & $400 \times 400$ & $0 \sim 10 \mathrm{~dB}$ \\
\hline
\end{tabular}

TABLE 5: Composition of test set.

\begin{tabular}{lccc}
\hline Data set & Test set & Size & Classes \\
\hline & & $400 \times 400$ & 1 \\
Different interpolating zero multiples & $100 / 100 / 100$ & $600 \times 600$ & 2 \\
& & $800 \times 800$ & 3 \\
Different number of blades & $20 / 20 / 20 / 20 / 20 / 20$ & $400 \times 400$ & $1 / 2 / 3 / 4 / 5 / 6$ \\
Different signal-to-noise ratio & $100 / 100 / 100$ & $400 \times 400$ & $0 / 3 / 10 \mathrm{~dB}$ \\
\hline
\end{tabular}

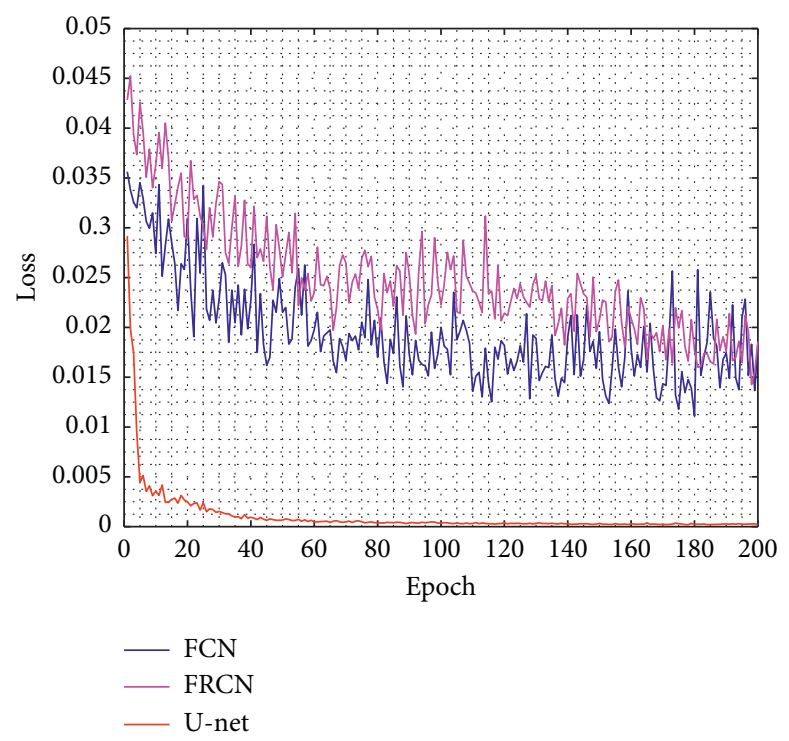

Figure 12: The loss curves of three networks during training. 


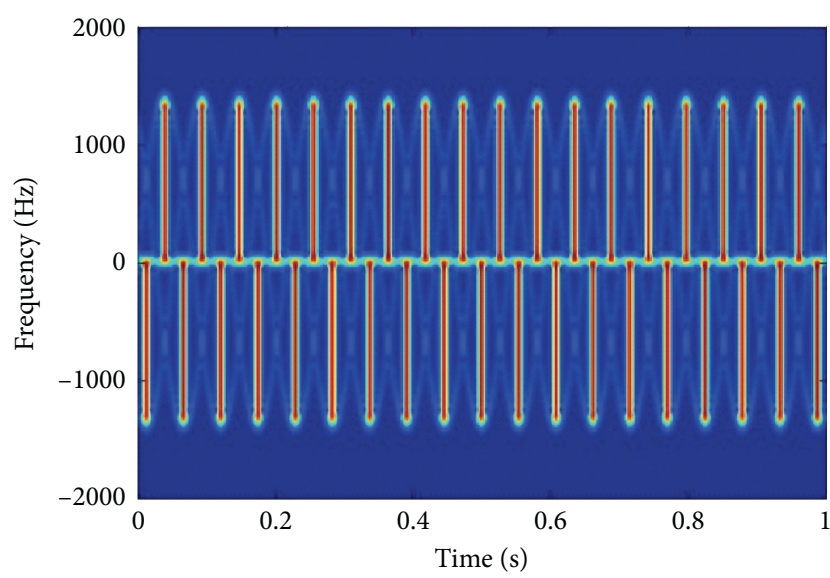

(a)

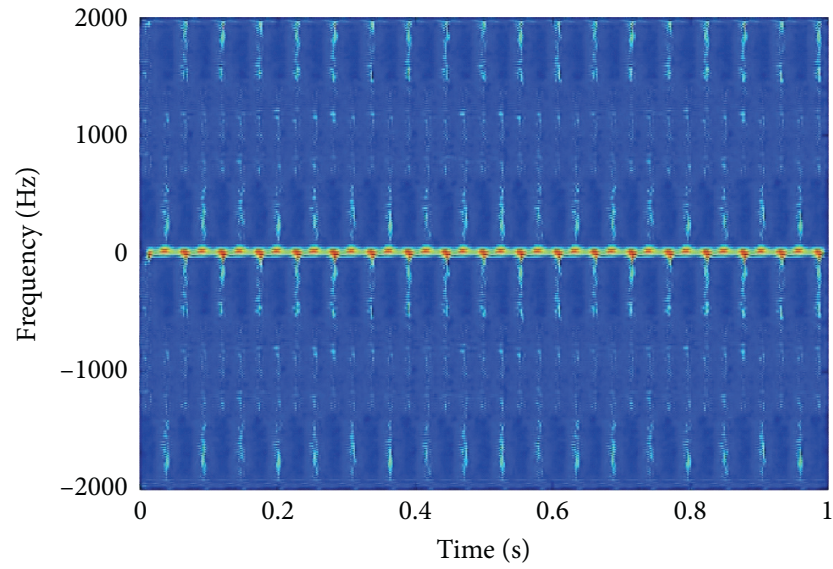

(c)

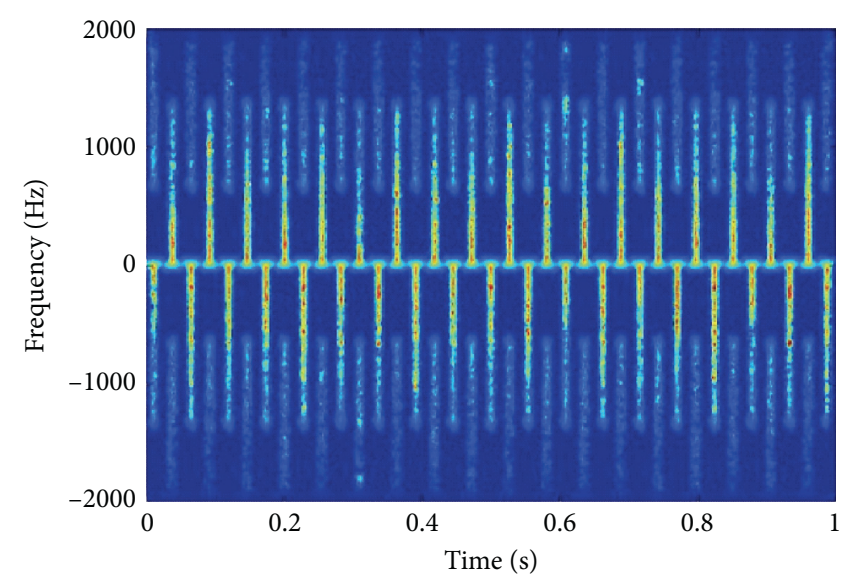

(b)

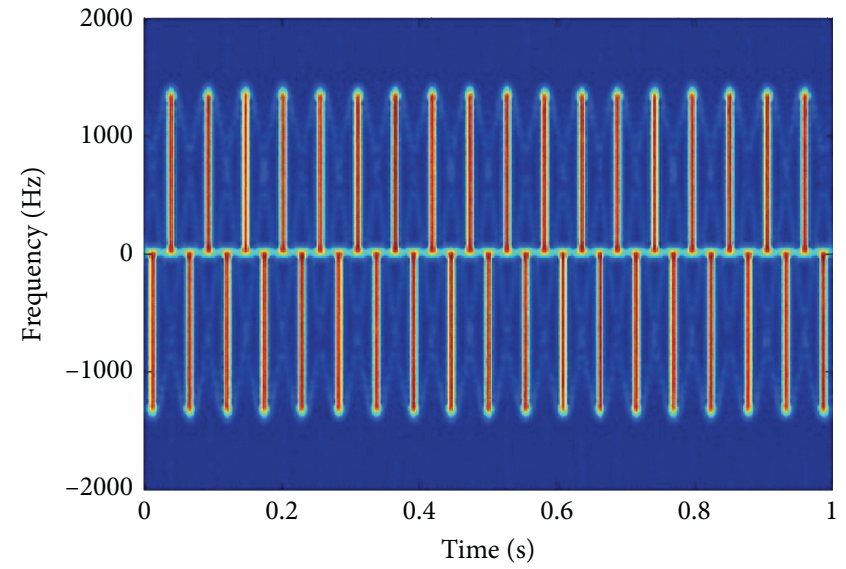

(d)

FIGURE 13: The outputs of the time-frequency results of the same input picture test with different models. (a) The label of the time-frequency results. The output of the time-frequency result with (b) FCN, (c) FCRN, and (d) U-net.

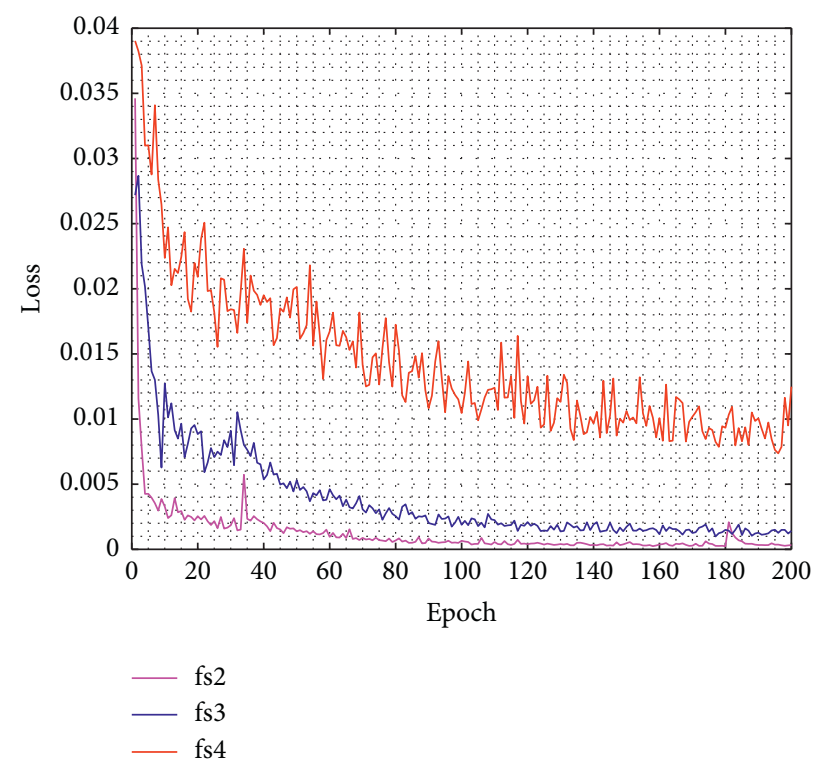

Figure 14: The loss curves of different zero interpolating multiples. 


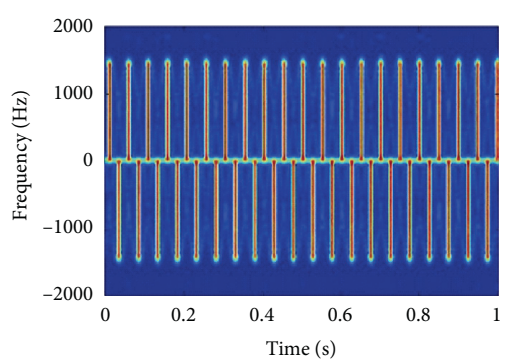

(a)

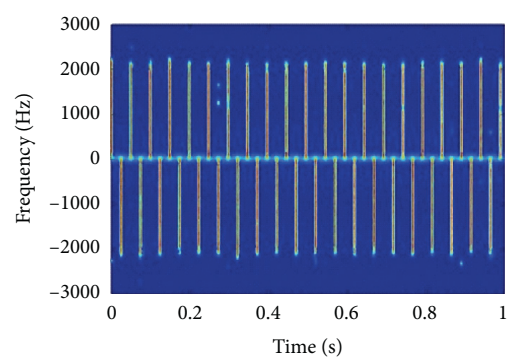

(b)

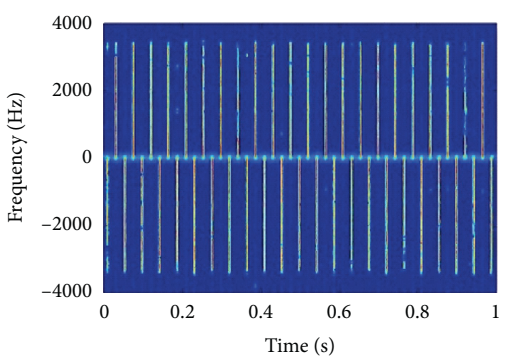

(c)

Figure 15: The outputs of time-frequency results of echo sequence after different interpolating zero multiples. The output of time-frequency result of echo sequence after interpolating (a) once of zeros, (b) double of zeros, and (c) triple of zeros.

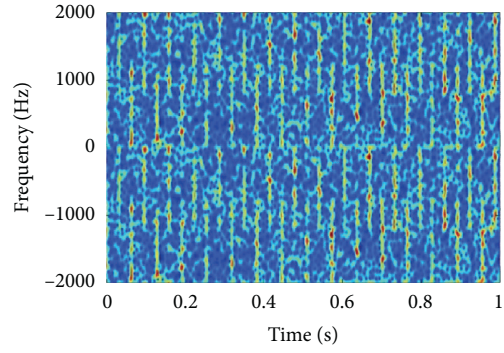

(a)

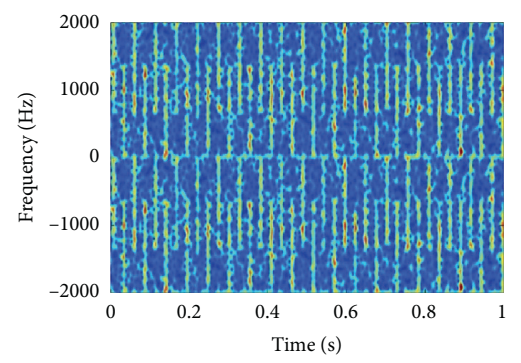

(b)

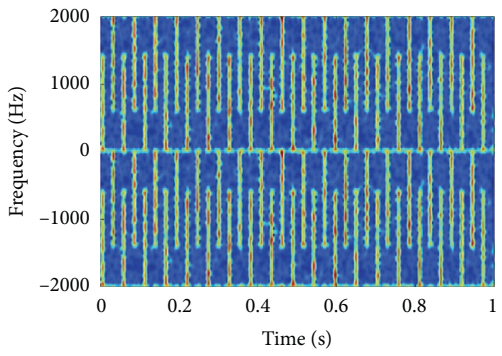

(c)

FIgURE 16: The input parts of the test set of time-frequency results with different SNRs. The test set of time-frequency result with (a) $\mathrm{SNR}=0 \mathrm{~dB}$, (b) $\mathrm{SNR}=3 \mathrm{~dB}$, and (c) $\mathrm{SNR}=10 \mathrm{~dB}$.

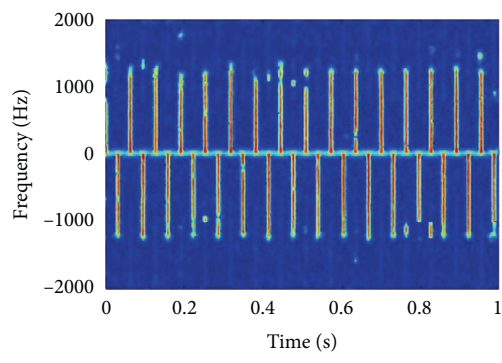

(a)

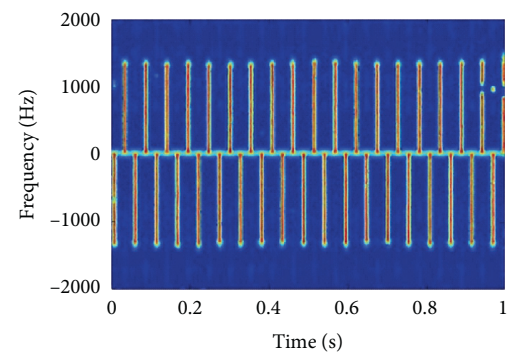

(b)

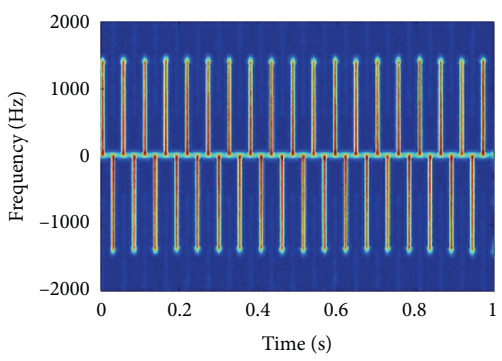

(c)

Figure 17: The outputs of time-frequency results with different SNRs. (a) SNR $=0 \mathrm{~dB}$. (b) SNR $=3 \mathrm{~dB}$. (c) SNR $=10 \mathrm{~dB}$.

similar. The performance of complex-valued U-net in the case of different number of blades is tested in this paper, and the training model is generated and tested by using the dataset of different number of blades. The results of timefrequency results of different number of blades are shown in
Figure 18. By comparing the input and output images of 1-6 blades in the test set, it can be found that the training model has a better capability in removing redundant flicker, and a large amount of useful information is retained in envelope preservation. 


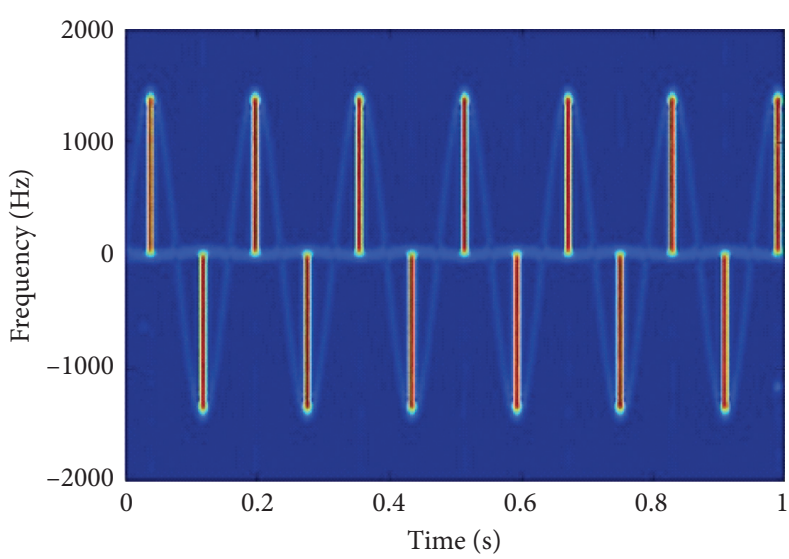

(a)

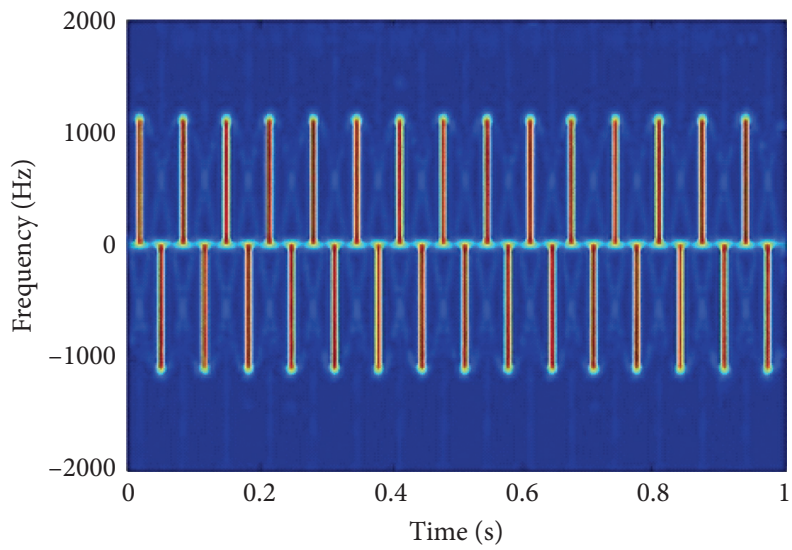

(c)

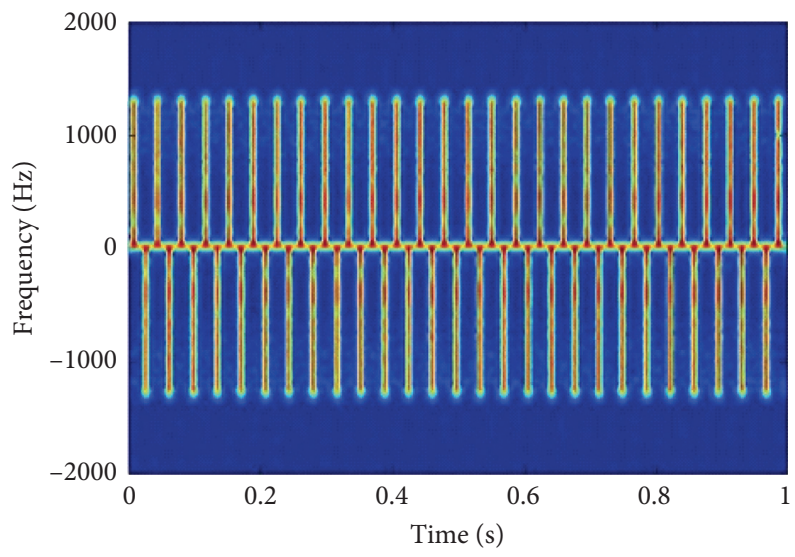

(e)

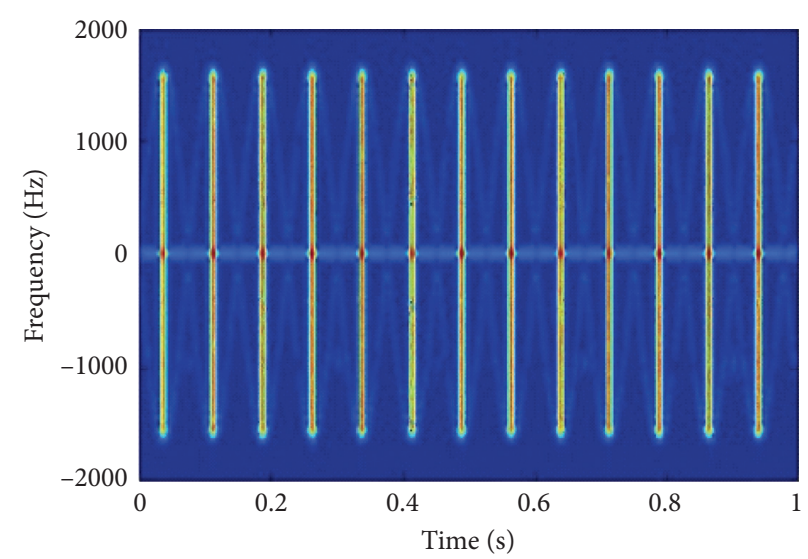

(b)

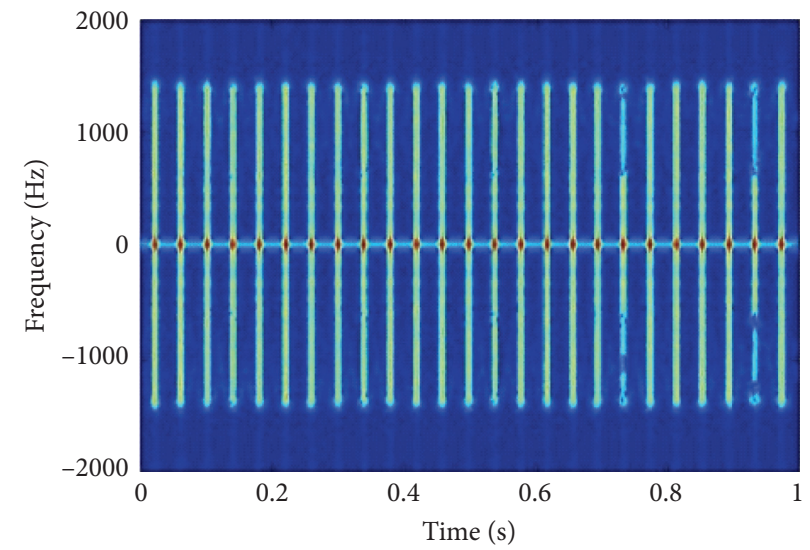

(d)

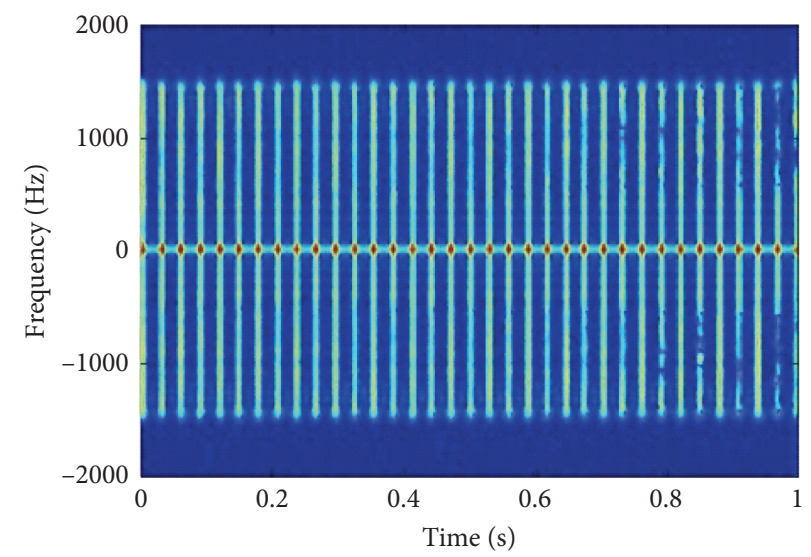

(f)

Figure 18: The results of time-frequency results of different number of blades. (a) One rotor. (b) Two rotors. (c) Three rotors. (d) Four rotors. (e) Five rotors. (f) Six rotors.

\section{Conclusions}

In order to resolute the problem of micro-Doppler frequency ambiguity, that is, the highest frequency in echo, which is higher than the sampling frequency, zero interpolation is proposed to increase the sampling frequency, and the complex-valued $U$-net is proposed to remove the redundant frequency components generated by zero interpolation after the real frequency is restored by zero interpolation. This method achieves the purpose of reconstructing the real micro-Doppler frequency.

Through the experimental analysis, the zero interpolation and complex-valued U-net can effectively solve the problem of micro-Doppler ambiguity in helicopter rotor echo. Compared with FCN and FCRN, the complex-valued U-net can effectively remove redundant flickers while 
retaining the required flicker and envelope information and has excellent performance to segment the time-frequency image of rotor targets as follows:

(1) It can remove the redundant frequency components generated by zero interpolation in different zero interpolating multiples. With the number of interpolation zeros increasing, it still has good performance.

(2) In a large signal-to-noise ratio range, it can effectively reduce the influence of noise on time-frequency results and remove the redundant frequency components generated by zero interpolation.

(3) For rotor targets, it can generate a training model to remove redundant frequency components generated by zero interpolation effectively for different number of rotor blades. The training model can save real flicker, and a large amount of useful information is retained in envelope preservation for feature extraction, parameter estimation, and recognition.

However, due to the limitation of experimental hardware and the amount of experimental data, the performance of the model is not optimal. The performance of the model will be tested by using the measured data for further research.

\section{Data Availability}

The numerical data used to support the findings of this study are included within the article.

\section{Conflicts of Interest}

The authors declare no conflicts of interest.

\section{Acknowledgments}

This research was funded by the National Natural Science Foundation of China under grant no. 61671469, the Key Project of the Army Equipment Department under grant KJ20201A020301, and "dual key" project.

\section{References}

[1] X. Yan, B. Lou, and A. Xie, "A review of advanced high-speed rotorcraft," Materials Science and Engineering, vol. 1102, Article ID 012006, 2021.

[2] A. Matus-Vargas, G. Rodriguez-Gomez, J. Martinez-Carranz, and J. Martinez-Carranza, "Ground effect on rotorcraft unmanned aerial vehicles: a review," Intelligent Service Robotics, vol. 14, no. 1, pp. 99-118, 2021.

[3] V. C. Chen, "Analysis of radar micro-Doppler signature with time-frequency transform," in Proceedings of the 10th IEEE Workshop on Statistical Signal and Array Processing, pp. 463-466, Pocono Manor, PA, USA, 2000.

[4] V. C. Chen, "Time-frequency signatures of micro-Doppler phenomenon for feature extraction," in Proceedings of SPIE in Wavlet Applications, vol. 4056, pp. 220-226, Orlando, FL, USA, 2000.

[5] G. E. Smith, K. Woodbridge, and C. J. Baker, "Micro-doppler signature classification," in Proceedings of the 2006 CIE
International Conference on Radar, pp. 1-4, Shanghai, China, 2006.

[6] G. Wang, S. Tan, C. Guan, N. Wang, and Z. Liu, "Multiple model particle filter track-before-detect for range ambiguous radar," Chinese Journal of Aeronautics, vol. 26, no. 6, pp. 1477-1487, 2013.

[7] D. K. A. Pulutan and J. S. Marciano, "Design trade-offs in a combined FMCW and pulse Doppler radar front-end," in Proceedings of the IEEE 2013 Tencon-Spring, pp. 567-571, Sydney, Australia, 2013.

[8] P. Iervolino, M. Cohen, R. Guida, and P. Whittaker, "Shipdetection in SAR imagery using low pulse repetition frequency radar," in Proceedings of the 10th European Conference on Synthetic Aperture Radar, pp. 1-4, Berlin, Germany, 2014.

[9] H. Dong, H. Chen, and J. Hui, "Broadband mid-infrared pulse via intra-pulse difference frequency generation based on supercontinuum from multiple thin plates," Chinese Physics $B$, vol. 28, Article ID 114203, 2019.

[10] E. Ewertowska, B. Mercadal, V. Muñoz, A. Ivorra, M. Trujillo, and E. Berjano, "Effect of applied voltage, duration and repetition frequency of RF pulses for pain relief on temperature spikes and electrical field: a computer modelling study," International Journal of Hyperthermia, vol. 34, no. 1, pp. 112-121, 2018.

[11] I. Long and H. William, Target Detection in a Medium Pulse Repetition Frequency Pulse Doppler Radar, 1978, https://www. freepatentsonline.com/4093948.pdf.

[12] I. V. Clarkson, S. Sirianunpiboon, and S. D. Howard, "Frequency estimation in coherent, periodic pulse trains," IEEE Signal Processing Letters, vol. 99, p. 1, 2020.

[13] Q. Liang, "Research and development of solid state high power high repetition frequency pulse source," Telecommunication Engineering, vol. 10, pp. 117-126, 2019.

[14] C. Ding, D. Pei, and A. Salomaa, "Chinese remainder theorem," Computing Coding Cryptography, vol. 224, 2005.

[15] W. Chen, Q. Yin, and H. Chen, "Robust Chinese remainder theorem ranging method based on dual-frequency measurements," IEEE Transactions on Vehicular Technology, vol. 60, pp. 4094-4099, 2011.

[16] Z. L. Lian, D. Z. Zhang, and X. J. Zhang, "Velocity ambiguity resolution based on look-up table method," Radar Science and Technology, vol. 8, pp. 70-73, 2011.

[17] L. Ming, M. Li, and Z. Lei, "A high efficient range ambiguity resolution algorithm for $\mathrm{PD}$ radar base on one-dimensional set," Proceedings of SPIE - The International Society for Optical Engineering, vol. 8285, pp. 170-177, 2011.

[18] V. C. Chen, F. Li, S.-S. Ho, and H. Wechsler, "Analysis of micro-Doppler signatures," Radar, Sonar and Navigation, vol. 150, no. 4, pp. 271-276, 2003.

[19] V. C. Chen, "Doppler signatures of radar backscattering from objects with micro-motions," IET Signal Processing, vol. 2, no. 3, pp. 291-300, 2008.

[20] V. C. Chen, "The Micro-Doppler Effect in Radar," Artech House, pp. 105-127, Norwood, MA, USA, 2011.

[21] J. Schmidhuber, "Deep learning in neural networks: an overview," Neural Networks, vol. 61, pp. 85-117, 2015.

[22] W. Ma, M. Papadakis, A. Tsakmalis, M. Cordy, and Y. L. Traon, "Test selection for deep learning systems," ACM Transactions on Software Engineering and Methodology, vol. 30, no. 2, pp. 1-22, 2021.

[23] I. Goodfellow, Y. Bengio, and A. Courville, Deep Learning, MIT Press, Cambridge, UK, 2016.

[24] R. Q. Charles, H. Su, M. Kaichun, and L. J Guibas, "Deep learning on point sets for 3D classification and segmentation," 
in Proceedings of the 2017 IEEE Conference on Computer Vision and Pattern Recognition, pp. 77-85, Honolulu, HI, USA, 2017.

[25] X. Hao, G. Zhang, and S. Ma, "Deep learning," International Journal of Semantic Computing, vol. 10, no. 3, pp. 417-439, 2016.

[26] L. Deng, Foundations and Trends in Signal Processing: Deep Learning - Methods and Applications, Springer Publishing Company, New York, NY, USA, 2014.

[27] Y. Bengio, "Learning deep architectures for AI," Foundations and Trends in Machine Learning, vol. 2, no. 1, pp. 1-127, 2009.

[28] S. Dong, P. Wang, and K. Abbas, "A survey on deep learning and its applications," Computer Science Review, vol. 40, p. 100379, 2021.

[29] O. Ronneberger, P. Fischer, and T. Brox, "U-net: convolutional networks for biomedical image segmentation," Lecture Notes in Computer Science, vol. 9351, pp. 234-241, Springer, Cham, Switzerland, 2015.

[30] J. Long, E. Shelhamer, and T. Darrell, "Fully convolutional networks for semantic segmentation," IEEE Transactions on Pattern Analysis and Machine Intelligence, vol. 39, pp. 640651, 2015.

[31] T. P. Van, S. T. Nguyen, and B. L. Doan, "Efficient palm-line segmentation with U-net context fusion module," in Proceedings of the 2020 International Conference on Advanced Computing and Applications, pp. 23-28, Quy Nhon, Vietnam, 2020.

[32] J. Chen, Y. Lu, Q. Yu et al., TransUNet: Transformers Make Strong Encoders for Medical Image Segmentation, 2021, https://arxiv.org/abs/2102.04306. 